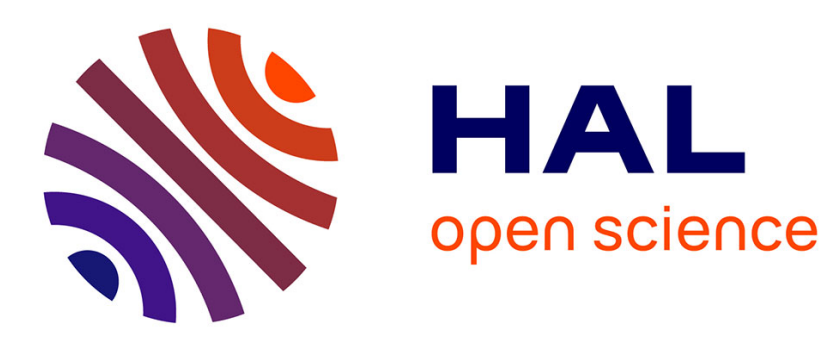

\title{
Towards a new method of porosimetry: principles and experiments
}

Antonio Rodriguez de Castro, Abdelaziz Omari, Azita Ahmadi-Sénichault, Denis Bruneau

\section{- To cite this version:}

Antonio Rodriguez de Castro, Abdelaziz Omari, Azita Ahmadi-Sénichault, Denis Bruneau. Towards a new method of porosimetry: principles and experiments. Transport in Porous Media, 2013, 101 (3), pp.349-364. 10.1007/s11242-013-0248-5 . hal-01174028

\section{HAL Id: hal-01174028 \\ https://hal.science/hal-01174028}

Submitted on 25 Oct 2017

HAL is a multi-disciplinary open access archive for the deposit and dissemination of scientific research documents, whether they are published or not. The documents may come from teaching and research institutions in France or abroad, or from public or private research centers.
L'archive ouverte pluridisciplinaire HAL, est destinée au dépôt et à la diffusion de documents scientifiques de niveau recherche, publiés ou non, émanant des établissements d'enseignement et de recherche français ou étrangers, des laboratoires publics ou privés. 


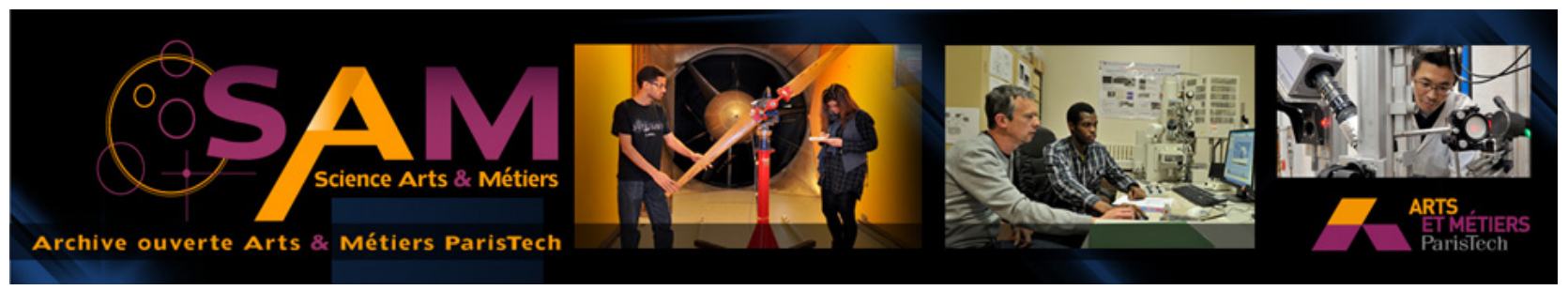

Science Arts \& Métiers (SAM)

is an open access repository that collects the work of Arts et Métiers ParisTech researchers and makes it freely available over the web where possible.

This is an author-deposited version published in: http://sam.ensam.eu

Handle ID: .http://hdl.handle.net/10985/9722

\section{To cite this version :}

Antonio RODRIGUEZ DE CASTRO, Aziz OMARI, Azita AHMADI, Denis BRUNEAU - Towards a new method of porosimetry: principles and experiments - Transport in Porous Media - Vol. 101, n`3, p.349-364 - 2013 


\title{
Toward a New Method of Porosimetry: Principles and Experiments
}

\author{
Antonio Rodríguez de Castro • Abdelaziz Omari • \\ Azita Ahmadi-Sénichault • Denis Bruneau
}

\begin{abstract}
Current experimental methods used to determine pore size distributions (PSD) of porous media present several drawbacks such as toxicity of the employed fluids (e.g., mercury porosimetry). The theoretical basis of a new method to obtain the PSD by injecting yield stress fluids through porous media and measuring the flow rate $Q$ at several pressure gradients $\nabla P$ was proposed in the literature. On the basis of these theoretical considerations, an intuitive approach to obtain PSD from $Q(\nabla P)$ is presented in this work. It relies on considering the extra increment of $Q$ when $\nabla P$ is increased, as a consequence of the pores of smaller radius newly incorporated to the flow. This procedure is first tested and validated on numerically generated experiments. Then, it is applied to exploit data coming from laboratory experiments and the obtained PSD show good agreement with the PSD deduced from mercury porosimetry.
\end{abstract}

Keywords Porosimetry · Yield stress · Experimental method · Pore size distribution · Porous media

\section{Introduction}

Porous media are inherent in many industrial applications such as oil recovery, soil remediation, $\mathrm{CO}_{2}$ underground storage, and geothermal energy storage. Due to their internal structure and their high surface to volume ratio inducing high performance in heat and mass transfer processes, they are used in numerous industrial processes namely those in fuel cells, paper pulp drying, filtration, etc. Therefore, continuously growing research efforts are devoted to study porous media structure and especially the flow of Newtonian fluids as well as complex fluids therein.

A. Rodríguez de Castro $(\varangle) \cdot$ A. Omari · A. Ahmadi-Sénichault · D. Bruneau TREFLE Department, Institut de Mécanique et d'Ingénierie de Bordeaux, Arts et Métiers ParisTech, Esplanade des Arts et Métiers, 33405 Talence, France e-mail: antonio.rodriguez@ensam.eu 
The structure of the porous space is complex with tortuous and interconnected pathways (Sun et al. 2013), and the use of global porosity $\epsilon$ (porous volume/total volume ratio) and permeability $K$ of porous media does not provide any information about local dimension and shape of the void space. Even if pore space is continuous, pores structure is often discretized as a succession of connected pore bodies and pore throats. However, a simple and widely used representation of pore space consists in a bundle of straight parallel capillaries with various sizes following a particular distribution, where a given pore radius $r$ is weighted by a probability factor $p(r)$. The shape of the pore size distribution (PSD) is determined by the geological history and composition of the material. Moreover, the PSD is linked to quantities such as capillary pressure, hydraulic conductivity, and solute dispersion (Nimmo 2004). Determination of the PSD is thus a routine task executed by means of several experimental methods.

Among these methods, stereology is a time consuming method which consists in deducing pore characteristics from imaging thin porous medium slices (Riche et al. 2012; Okabe and Blunt 2005; Galaup et al. 2012). Treatment and correlation of many successive images is needed to provide statistically representative shapes and sizes of the pore space. Others disadvantages of the thin sectioning method include the extensive man hours required in polishing/slicing/digitalization, the destructive nature of the technique and the fact that the digitalization resolution within each slice is much superior to the slice thickness (Lindquist and Venkatarangan 2000).

More recently, great progress on direct measurement of the pore space topology has been achieved with X-ray computed tomography (Lindquist and Venkatarangan 2000; Prodanovic et al. 2006, 2007). This nondestructive technique has a resolution of typically $1 \mu \mathrm{m}$, and a preset network model is needed in order to define a pore geometry. Regarding this definition of the pore geometry, several criteria are possible: spheres connected by channels, intersecting spheres, etc. The input of these networks include coordination number of pores, pore size distribution, pore throat distribution, pore body-to-pore throat aspect ratio, and pore body-to-pore body aspect ratio. Therefore, different networks may be obtained for the same sample as a consequence of the different possibilities to define pore bodies and pore throats (Prodanovic et al. 2006; Okabe and Blunt 2005). An excellent review of the tomography techniques and the acquisition of the pore structure from the images was presented by Plougonven (2009).

Due to the limitations, representativeness and cost of the techniques mentioned above, methods of invading fluids are favored even if each of them has some specific shortcomings. Water vapor adsorption methods have shortcomings with respect to the analysis of coarse size pores due to the lack of capillary condensation (Kate and Gokhale 2006). Low-pressure adsorption of $\mathrm{CO}_{2}$ is useful for characterizing microporosity (pore diameters $<2 \mathrm{~nm}$ ), while low-pressure adsorption of nitrogen is useful for characterizing meso and macroporosity (pore sizes between 2 and $50 \mathrm{~nm}$, and $>50 \mathrm{~nm}$, respectively) (Clarkson et al. 2013). Unfortunately, low-pressure adsorption has an upper pore diameter limit of about $300 \mathrm{~nm}$. Furthermore, this technique requires drying of the porous media samples to remove volatiles prior to analysis.

The mercury invasion porosimetry (MIP) consists in immersing a piece of porous medium in mercury and measuring the volume of the invading mercury versus the applied pressure, which gives the distribution of the capillary pressure as it represents the counterpart of the actually applied pressure (Giesche 2006). Also, knowing the mercury/solid interfacial tension and the contact angle, this data allows determination of pore size distribution using a modified Laplace equation referred to as Washburn equation. For that purpose the porous medium is usually modeled as a bundle of cylindrical capillaries, which leads to an overestimation of 
the occurrence of small pores. Indeed, the volume of the large pores situated downstream a constriction, which are filled at higher pressures than those predicted with Washburn equation, is assigned to the smaller pores. Moreover, the major shortcoming of MIP is the use of mercury, known to be a highly toxic fluid. Besides, a legally binding limitation on the use of mercury is being adopted (UNEP 2013). The IUPAC Working Group on Liquid Intrusion and Alternative Methods for the characterization of macroporous materials recently published a document (Rouquerol et al. 2012) with the intention of listing, examining, and comparing the methods presently used to characterize macroporous structures (pore width $>50 \mathrm{~nm}$ ). The conclusion was that none of the current alternatives is in a position to replace the mercury porosimetry. Indeed, none of them can provide in $2-3 \mathrm{~h}$ comparable information over so broad a pore sizes range. Therefore, abandoning mercury porosimetry would result in a loss of efficiency of research and of routine work on macroporous materials. It is then of great interest to develop new alternative methods.

In that respect, the aim of the present work is to propose a method to derive PSD from experiments of injection of a yield stress fluid in porous media. By measuring the flow rate as a function of the pressure gradient $Q(\nabla P)$ and through application of an intuitive treatment, the PSD can be deduced. In the following section the theoretical basis of the involved method is presented. Section 3 is devoted to the presentation of details concerning the procedures of raw data treatment and determination of the pore size distribution. Tests of the method on numerically generated experiments and on laboratory experiments are then presented and discussed in Sect. 4 before drawing some concluding remarks and perspectives.

\section{Theoretical Background}

Yield stress fluids are mainly characterized by a threshold stress $\tau_{0}$ below which they do not flow. The rheological behavior under shear of such fluids is mostly described by the Herschel-Bulkley law:

$$
\begin{aligned}
& \tau=\tau_{0}+k \dot{\gamma}^{n} \text { for } \tau \geq \tau_{0} \\
& \dot{\gamma}=0 \text { for } \tau \leq \tau_{0}
\end{aligned}
$$

where $k$ is the fluid consistency and $n$ its flow index. In other respect, for a fluid flowing through a capillary, the momentum balance under equilibrium state leads to a linear dependence of the shear stress on the radial coordinate $x$ for a given pressure gradient $\nabla P$ :

$$
\tau(x)=\frac{\nabla P x}{2} .
$$

Consequently, if a Herschel-Bulkley fluid is subjected to a pressure gradient $\nabla P$ and no-slip condition on the wall is assumed, no flow will occur in the capillaries whose radius is below the critical value $r_{\mathrm{c}}$ :

$$
r_{\mathrm{c}}=\frac{2 \tau_{0}}{\nabla P}
$$

Hence, the flow rate of a Herschel-Bulkley fluid through a capillary with an arbitrary radius $r$ is given by (Skelland 1967): 


$$
\begin{aligned}
& q(\nabla P, r)=\left[1-2\left[\frac{\left(1-\frac{2 \tau_{0}}{\nabla P r}\right)^{2}}{\frac{1}{n}+3}+\frac{\frac{2 \tau_{0}}{\nabla P r}\left(1-\frac{2 \tau_{0}}{\nabla P r}\right)}{\frac{1}{n}+2}\right]\right] \\
& \times\left[\frac{r}{\frac{1}{n}+1}\left(\frac{r \nabla P}{2 k}\right)^{\frac{1}{n}}\left(1-\frac{2 \tau_{0}}{\nabla P r}\right)^{\frac{1}{n}+1}\right] \pi r^{2} \text { for } \nabla P \geq 2 \tau_{0} / r \\
& q(\nabla P, r)=0 \text { for } \nabla P \leq 2 \tau_{0} / r .
\end{aligned}
$$

Now, if the porous medium is assumed to be well represented by a bundle of parallel capillaries of various radii, the total flow rate $Q$ for a given $\nabla P$ is related to $q(\nabla P, r)$ through the following relationship:

$$
Q\left(\nabla P_{j}\right)=\int_{0}^{\infty} q\left(\nabla P_{j}, r\right) p(r) \mathrm{d} r
$$

$p(r)$ being the pore size distribution function. In practice, the upper limit of the integral is finite and is equal to the radius of the largest pores $r_{\max }$, and the lower limit correspond to the smallest pore radius that participates in the flow $r_{\min }\left(\nabla P_{j}\right)=\frac{2 \tau_{0}}{\nabla P_{j}}$ at the involved pressure gradient $\nabla P_{j}$. Moreover when all pores are conducting, the overall flow rate at high pressure gradients is a power law of $\nabla P$ with an exponent of $1 / n$. As $q(\nabla P, r)$ encompasses the rheological characteristics of the fluid, an inversion procedure of the integral equation above leads to the determination of the pore size distribution. In their original work, Ambari et al. (1990) studied the simple case of a Bingham fluid $(n=1)$. Chaplain et al. (1992) extended such work to a bundle of randomly oriented capillaries and more recently, Oukhlef et al. (2011) considered the case of Herschel-Bulkley fluids. They showed that the analytical inversion of Eq. (5) leads to the following expression of $p(r)$ :

$$
p(r)=\left.\frac{2^{\frac{1+3 n}{n}} k^{1 / n} \nabla P^{2}}{16(1 / n !) \pi \tau_{0} r^{\frac{1+3 n}{n}}}\left[\left(\frac{1+4 n}{n}\right) \frac{\partial^{\left(\frac{1+n}{n}\right)}}{\partial \nabla P^{\left(\frac{1+n}{n}\right)}}+\nabla P \frac{\partial^{\left(\frac{1+2 n}{n}\right)}}{\partial \nabla P^{\left(\frac{1+2 n}{n}\right)}}\right] Q\right|_{\nabla P=\frac{2 \tau_{0}}{r}} .
$$

If the value of $1 / n$ is not an integer, the last equation involves the calculation of fractional derivatives, which may be complex in some cases (Weilbeer 2005).

In order to test this method, Oukhlef (2011) performed in silico experiments of a HerschelBulkley fluid flowing through a bundle of capillaries with a pre-defined PSD. A polynomial function was then used to fit at best the raw numerical data $(Q, \nabla P)$ and $p(r)$ was calculated using the Eq. (6). The obtained results were then compared to the original size distribution. He showed the method to work very well in the case of Bingham fluids $(n=1)$. However when $n \neq 1$ the procedure is less convincing since high fluctuations are noticed at low radii and the quality of the obtained results is reported to depend on the degree of the polynomial function chosen for the fitting step in the procedure (see Oukhlef et al. 2011 for details).

\section{Description of the Present Method}

Like in standard mercury porosimetry, and as in the analytical method presented in the previous section, the porous medium is modeled as a bundle of capillaries whose radii are distributed according to a probability density function $p(r)$ and hence, the flow rate through a class of pores of radius $r_{i}, q\left(\nabla P, r_{i}\right)$, is given by Eq. 4 for $r=r_{i}$. During a typical experiment, 
a yield stress fluid whose rheology is described by the Herschel-Bulkley model (Eq. 1) is injected through the investigated porous medium and a set of $N+1$ raw data $\left(Q_{i}, \nabla P_{i}\right)$ with $i=(1, \ldots, N+1)$ are collected and arranged in an ascending manner. The range of the data should be so that the lowest flow rate is as close to zero as possible and the upper flow rate lies in the interval where $Q(\nabla P)$ follows the power law $\nabla P^{y}$ with $y$ close to $1 / n$. Each $\nabla P_{i}$ defines a class of pores of representative radius $r_{i}$ taken here as being $r_{i}=\alpha \frac{2 \tau_{0}}{\nabla P_{i}}$ $\left(r_{1}>r_{2}>\cdots>r_{N}\right)$ where $\alpha$ is a factor greater than unity whose precise value will be discussed later. Besides, rather than using Eq. (6) to determine the PSD, we simply express that the measured flow rate at a given $\nabla P_{j}$ is greater or equal to the sum of the contributions of all the pores whose radii are larger than $r_{j}$. So it can be written $Q_{j} \geq \sum_{i=1}^{j-1} n_{i} q\left(\nabla P_{j}, r_{i}\right)$, where $n_{i}$ represents the number of pores of each $i$ th class. In order to obtain the PSD, the $N$ unknowns $n_{i}$ must be determined by solving the following system of inequalities:

$$
\begin{aligned}
Q_{2} & \geq n_{1} q\left(\nabla P_{2}, r_{1}\right) \\
Q_{3} & \geq n_{1} q\left(\nabla P_{3}, r_{1}\right)+n_{2} q\left(\nabla P_{3}, r_{2}\right) \\
\quad & \\
Q_{j} & \geq n_{1} q\left(\nabla P_{j}, r_{1}\right)+n_{2} q\left(\nabla P_{j}, r_{2}\right)+\cdots+n_{j-1} q\left(\nabla P_{j}, r_{j-1}\right) \\
\quad & \\
Q_{N+1} & \geq n_{1} q\left(\nabla P_{N+1}, r_{1}\right)+n_{2} q\left(\nabla P_{N+1}, r_{2}\right)+\cdots+n_{N} q\left(\nabla P_{N+1}, r_{N}\right) .
\end{aligned}
$$

These inequalities express the fact that the measured flow rate at a given $\nabla P_{j}$ should be greater or equal to the one calculated if only pores of radii larger than $r_{j}$ are taken into account. In the routine used to calculate a targeted $n_{i}$, and starting with $n_{1}$, successive and increasing positive values are scanned until one of the inequalities is violated and the maximum value is assigned to $n_{i}$. In this procedure, and as large pores contribute significantly to the total flow rate, the scanning should be more refined when the considered pore radius is large. Once $n_{i}(i=1, \ldots, N)$ have been calculated, the probability (in terms of relative volume) $p\left(r_{i}\right)$ is obtained through:

$$
p\left(r_{i}\right)=\frac{n_{i} \pi r_{i}^{2}}{\sum_{j=1}^{N} n_{j} \pi r_{j}^{2}} \text { for } i=1, \ldots, N .
$$

Here, it has been set that the representative radius of a class of pores $r_{i}$ is linked to $\nabla P_{i}$ through $r_{i}=\alpha \frac{2 \tau_{0}}{\nabla P_{i}}$ where $\alpha$ is a factor greater or equal to one. The fundamental reason of this is that for a given $\nabla P_{i}$ the resulting $Q_{i}$ is due to the pores whose radii are strictly larger than $\frac{2 \tau_{0}}{\nabla P_{i}}$. In other words, $\nabla P_{i}$ represents only the onset of contribution to the flow of pores of radius $\frac{2 \tau_{0}}{\nabla P_{i}}$. To determine the suitable value of $\alpha$, which will be named $\alpha^{*}$, successive values will be used to solve the system (7), starting with $\alpha=1$ and in increasing order until the following condition is satisfied:

$$
\begin{aligned}
& \exists s<N \text { such that } n_{i}=0 \quad \forall i>s \\
& \text { and } \exists f \in[s+1, N] \text { such that } Q_{f}=\sum_{g=1}^{s} n_{g} q\left(\nabla P_{f}, r_{g}\right)
\end{aligned}
$$

where $s, f$ and $g$ are integers.

What is expressed in condition (9) is that, as it has already been said, the last measures should correspond to $(Q, \nabla P)$ couples assuring conduction in all pore classes. Therefore, 
no further contribution of any pore class to the flow is expected at least between the last two $(Q, \nabla P)$ couples. Also, the last inequality in (7) has to be violated at the same moment as another previous one (the $f$-est), which indicates that there is no more extra growth of the total flow rate due to newly incorporated pores from $\left(Q_{f}, \nabla P_{f}\right)$ to $\left(Q_{N+1}, \nabla P_{N+1}\right)$. This last condition (9) allows to determine the correct value of $\alpha$, i.e., $\alpha=\alpha^{*}$. In the next section it will be shown how the value of $\alpha$ influences the representativeness of the obtained PSD.

\section{Test of the Method}

In this part we successively test the method presented in the preceding section in the case of numerical experiments and in the case of laboratory experiments carried out on porous medium with unknown PSD.

\subsection{Numerical Experiments}

\subsubsection{Experimental Procedure}

A yield stress fluid, whose yield stress, consistency and flow index are respectively $\tau_{0}=$ $10 \mathrm{~Pa}, k=1 \mathrm{Pas}^{n}$ and $n=0.60$ was injected numerically through a bundle of $1,000,000$ capillaries whose radii are distributed according to the following bimodal probability density function

$$
p(r)=\frac{w_{1}}{\sqrt{2 \pi \sigma_{1}^{2}}} \mathrm{e}^{-\frac{\left(r-\mu_{1}\right)^{2}}{2 \sigma_{1}^{2}}}+\frac{w_{2}}{\sqrt{2 \pi \sigma_{2}^{2}}} \mathrm{e}^{-\frac{\left(r-\mu_{2}\right)^{2}}{2 \sigma_{2}^{2}}}
$$

which is a weighted sum of two normal laws with means $\mu_{1}=12 \mu \mathrm{m}$ and $\mu_{2}=24 \mu \mathrm{m}$, standard deviations, $\sigma_{1}=3 \mu \mathrm{m}$ and $\sigma_{2}=6 \mu \mathrm{m}$, and weights $w_{1}=2 / 3$ and $w_{2}=1 / 3$.

50 different pressure gradients $(N+1=50)$ given by $\nabla P_{i}=\frac{2 \tau_{0}}{z_{i}}$ with $i=(0, \ldots, N)$, $z_{i}=z_{\max }-\frac{\left(z_{\max }-z_{\min }\right) i}{N}, z_{\min }=\mu_{1}-3 \sigma_{1}$, and $z_{\max }=\mu_{2}+3 \sigma_{2}$ were considered and the resulting flow rates were calculated using Eq. (5). The obtained data are displayed in Fig. 1.

\subsubsection{Pore Size Distribution}

The method presented in Sect. 3 was applied to the $\left(Q_{i}, \nabla P_{i}\right)$ data and $\alpha^{*}$ satisfying condition (9) was found to be 1.05. The calculated PSD in terms of $n_{i}$ (number of pores of radius $r_{i}$ ) is presented in Fig. 2 together with the original PSD. It can be observed that the calculated PSD agrees very well with the original PSD. Furthermore, in order to study the influence of the used value of $\alpha$, the PSDs corresponding to several values of $\alpha$ were calculated and compared to the original PSD as shown in Fig. 3 for some of these values. From this figure it can be observed that the obtained PSDs globally capture the correct form of the original PSD. The agreement is qualitatively worse with $\alpha=1$. Moreover, when $\alpha>1.05$ the calculated PSDs also deviate more or less from the original PSD and $\alpha=\alpha^{*}=1.05$ is the best value in the present case. To go further, these values of $\alpha$ were tested in the case of different sets of distribution parameters $\mu_{1}, \mu_{2}, \sigma_{1}, \sigma_{2}, w_{1}$ and $w_{2}$, and the deviations from the original PSD were evaluated by calculating the following quantity $d(\alpha)$ :

$$
d(\alpha)=\sum_{i=1}^{N}\left|\frac{n_{i o}}{\sum_{i=1}^{N} n_{i o}}-\frac{n_{i}(\alpha)}{\sum_{i=1}^{N} n_{i}(\alpha)}\right|
$$




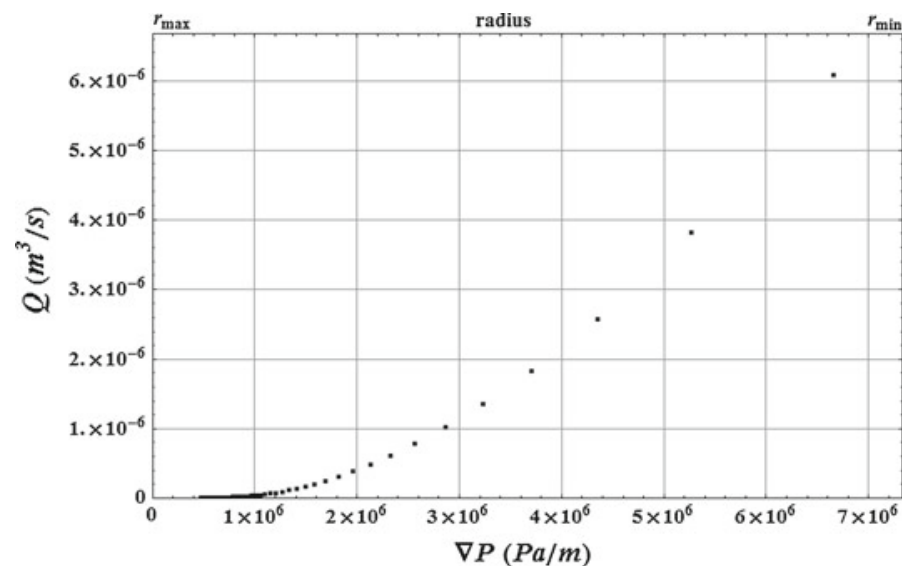

Fig. $1\left(Q_{i}, \nabla P_{i}\right)$ data corresponding to the numerical injection of a yield stress fluid with $\tau_{0}=10 \mathrm{~Pa}$, $k=1 \mathrm{Pas}^{n}$ and $n=0.60$ through a bundle of $1,000,000$ capillaries whose radii are distributed according to the given $p(r)$. The upper axis represents the radii of the pores joining the flow at each $\nabla P$ (smaller pores at high $\nabla P$ )

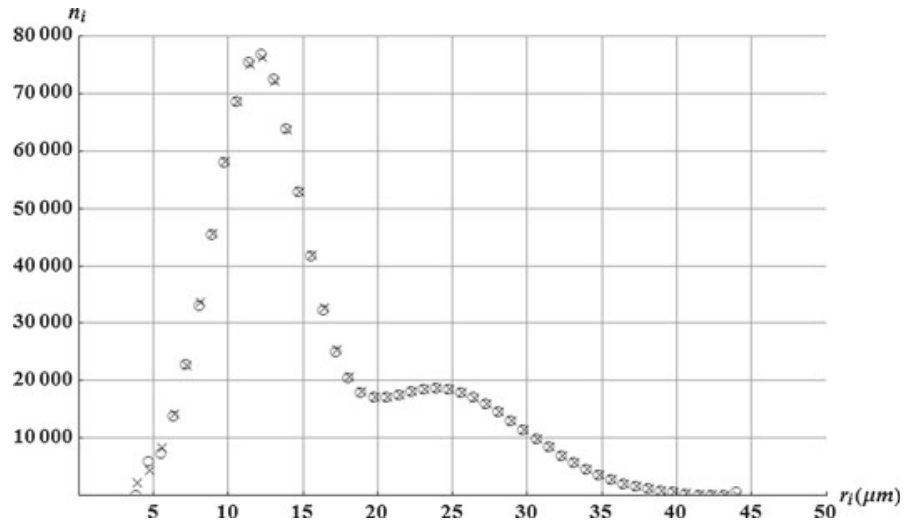

Fig. 2 Calculated PSD (circles) versus original PSD (crosses) corresponding to the numerical experiment here presented, for which $\alpha^{*}=1.05$

where $N+1$ is the number of data $(N=49), n_{i o}$ is the number of pores of radius $r_{i}$ corresponding to the original PSD (after discretization), $n_{i}(\alpha)$ is the number of pores of radius $r_{i}$ for the given $\alpha$, and $\sum_{i=1}^{N} n_{i o}$ is the total number of pores $(1,000,000$ in this case). The value of $d(\alpha)$ is bounded below by zero (no difference) and above by two. The obtained deviations as a function of $\alpha$ are tabulated in Table 1, where it is observed that whatever the considered distribution, the value of $\alpha^{*}$ obtained by applying the method is approximately the same (1.05). Moreover, when $\alpha$ is varied, best PSD are obtained for $\alpha>1$ and those corresponding to $\alpha$ far from the $\alpha^{*}$ given by the method (1.05) are less satisfactory.

\subsection{Laboratory Experiments}

\subsubsection{Materials}

The fluid to be injected is a xanthan gum solution obtained by dissolving an amount of xanthan gum powder in de-ionized and filtered water containing $400 \mathrm{ppm}$ of $\mathrm{NaN}_{3}$ as a bactericide. 
(a)

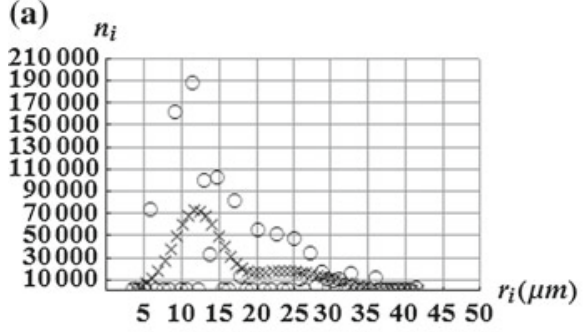

(c)

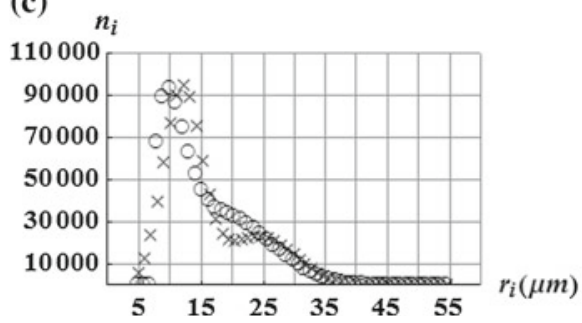

(b) $n_{i}$

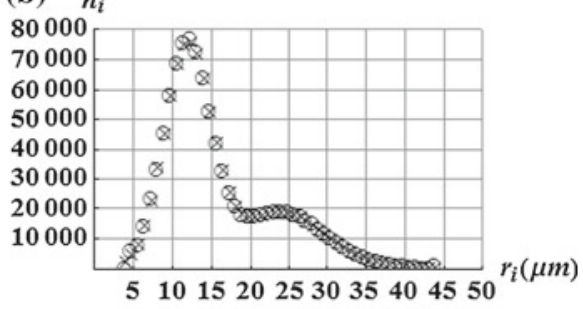

(d)

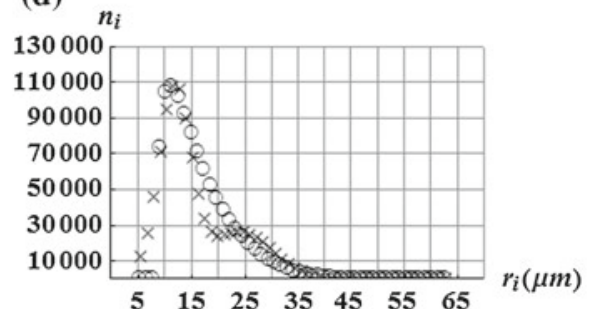

Fig. 3 Calculated PSD (circles) versus original PSD (crosses) corresponding to the numerical experiment here presented. $\mathbf{a} \alpha=1, \mathbf{b} \alpha=\alpha^{*}=1.05, \mathbf{c} \alpha=1.3$ and $\mathbf{d} \alpha=1.5$

Table 1 Deviation obtained for a set of experiments as a function of $\alpha$. The fluid is always the same $\left(\tau_{0}=\right.$ $10 \mathrm{~Pa}, k=1 \mathrm{Pas}^{n}$, and $n=0.60$ ) for different PSDs given by Eq. (10)

\begin{tabular}{llllllll}
\hline$\mu_{1}(\mu \mathrm{m})$ & 12 & 12 & 6 & 18 & 12 & 12 & 12 \\
$\mu_{2}(\mu \mathrm{m})$ & 24 & 24 & 48 & 21 & 24 & 24 & 24 \\
$\sigma_{1}(\mu \mathrm{m})$ & 3 & 3 & 3 & 3 & 1.5 & 3.5 & 3 \\
$\sigma_{2}(\mu \mathrm{m})$ & 6 & 6 & 6 & 6 & 3 & 8 & 6 \\
$w_{1}$ & $2 / 3$ & $1 / 3$ & $2 / 3$ & $2 / 3$ & $2 / 3$ & $2 / 3$ & 1 \\
$w_{2}$ & $1 / 3$ & $2 / 3$ & $1 / 3$ & $1 / 3$ & $1 / 3$ & $1 / 3$ & 0 \\
$d(\alpha=1)$ & 1.11 & 0.98 & 1.35 & 1.29 & 1.23 & 1.49 & 1.13 \\
$d(\alpha=1.05)$ & 0.01 & 0.01 & 0.07 & 0.02 & 0.03 & 0.09 & 0.01 \\
$d(\alpha=1.10)$ & 0.03 & 0.03 & 0.16 & 0.06 & 0.12 & 0.03 & 0.04 \\
$d(\alpha=1.30)$ & 0.29 & 0.51 & 1.33 & 0.27 & 0.49 & 0.26 & 0.30 \\
$d(\alpha=1.50)$ & 0.27 & 0.60 & 1.33 & 0.30 & 0.60 & 0.30 & 0.61 \\
$\alpha^{*}$ from condition $(9)$ & 1.05 & 1.05 & 1.05 & 1.05 & 1.05 & 1.10 & 1.05 \\
\hline
\end{tabular}

The concentration of xanthan gum solution $C_{\mathrm{p}}$ was alternatively fixed at 6,000 or 7,000 ppm depending on the experiment. Xanthan gum is a commonly used viscosifier (Garcia-Ochoa et al. 2000; Abidina et al. 2012) especially in manufacturing food grade products (Palaniraj and Jayaraman 2011), and is obtained through fermentation of Xanthomonas campestris bacteria (Wadhai and Dixit 2011; Garcia-Ochoa et al. 2000; Palaniraj and Jayaraman 2011). Its chemical composition, structure and other physico-chemical properties may be found elsewhere (Song et al. 2006). Its semi dilute aqueous solutions are known to develop a high viscosity level at low shear rates before displaying a drastic decrease when the applied shear rate is increased. Therefore, xanthan gum solutions are sometimes classified as yield stress fluids (Song et al. 2006; Khodja 2008) even if strictly speaking, they should be referred to 


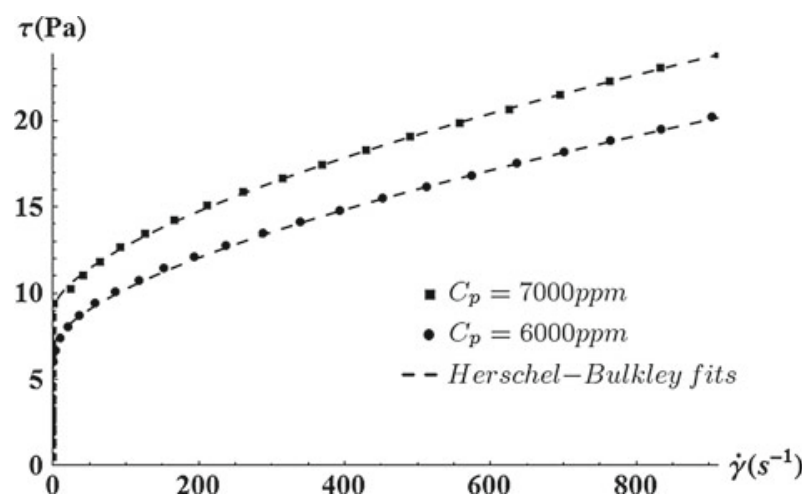

Fig. 4 Rheograms of the xanthan gum solutions used in laboratory experiments and their fits by a HerschelBulkley law. $C_{\mathrm{p}}=7,000 \mathrm{ppm}: \tau_{0}=9.04 \mathrm{~Pa}, k=0.21 \mathrm{Pas}^{n}, \quad n=0.63 . C_{\mathrm{p}}=6000 \mathrm{ppm}: \tau_{0}=$ $6.86 \mathrm{~Pa}, k=0.20 \mathrm{Pas}^{n}, n=0.62$

as pseudo-yield stress fluids. In solution state, an isolated xanthan macromolecule is more or less rigid and is of typically $1 \mu \mathrm{m}$ of contour length (Mongruel and Cloitre 2003) and a transverse size of approximately $2 \mathrm{~nm}$. In the case of porous media whose largest pores are larger than $1 \mu \mathrm{m}$, no plugging effect is expected at low $\nabla P$ as only large pores are involved in the flow. Under high $\nabla P$ the shear rate $\dot{\gamma}$ may be so high that the xanthan molecules are oriented in the direction of the flow and the limiting pore size that can be investigated should be compared to the transverse dimension of the molecules.

These polymer solutions were then characterized by means of a stress controlled rheometer (ARG2 from TA Instruments, France) equipped with a cone-plate geometry. Each shear rate corresponding to a specified shear stress was collected when its variation was less than a prescribed tolerance (typically $3 \%$ ) within a given time period. The obtained rheograms are displayed on Fig. 4 showing the shear stress versus shear rate. As can be observed the rheology is well fitted by a Herschel-Bulkley model for each xanthan concentration. The fitting procedure used here consists in minimizing the sum

$$
\sum_{i}\left(\tau\left(\dot{\gamma}_{i}\right)-\tau_{i}\right)^{2} \cdot \dot{\gamma}_{i}
$$

where $\tau(\dot{\gamma})$ is given by Eq. (1) and $\left(\dot{\gamma}_{i}, \tau_{i}\right)$ are experimental data, allowing determination of values of the yield stress $\tau_{0}$, the flow index $n$, and the consistency $k$ for each $C_{\mathrm{p}}$ as specified in the figure caption. The square of the difference between $\tau\left(\dot{\gamma}_{i}\right)$ and $\tau_{i}$ is weighted by $\dot{\gamma}_{i}$ in order to ensure a good fit for high shear rates while obtaining a convenient yield stress. Expression (12) was evaluated in both cases $(6,000$ and 7,000 ppm) resulting in an approximately $50 \%$ greater value for $6,000 \mathrm{ppm}$. Therefore, and as it is expected, the higher the polymer concentration, the better is the quality of the Herschel-Bulkley fit.

Again, it has to be noted that xanthan gum solutions are not actually yield stress fluids (if yield stress fluids do really exist). However, they present a Newtonian plateau of very high viscosity at low shear rates and a drastical shear-thinning behavior (the plateau viscosity of the $7,000 \mathrm{ppm}$ solution is approximately $10^{4}$ times its viscosity at $100 \mathrm{~s}^{-1}$ ). For that reason, and as a first approximation, their rheology may be well fitted by a Herschel-Bulkley model (Sochi 2010). Some authors claim that this procedure leads to arbitrary values of the yield stress and that other methods might be used to obtain the correct value of the yield stress (Nguyen and Boger 1983; Balhoff et al. 2011). Moreover, the rheology of these solutions may 


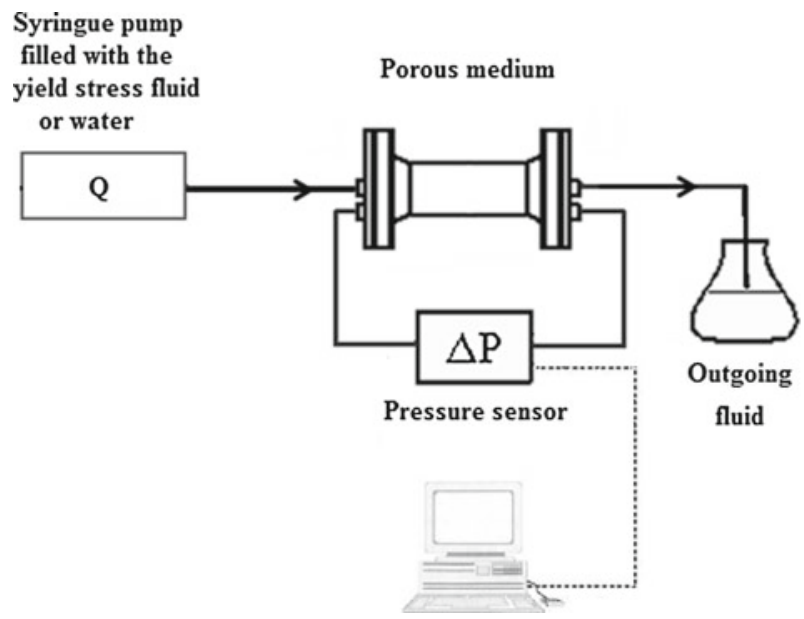

Fig. 5 Schematic representation of the experimental setup

be also well fitted by using the popular Carreau model, but unfortunately no rigorous analytic relation expressing $Q$ versus $\nabla P$ in a capillary exists (Balhoff et al. 2011, 2012). Therefore, the use of an asymptotic rheological law (truncated power law) should result in errors of the same order as those introduced by the Herschel-Bulkley model. Nevertheless, from a practical point of view, the most important issue in this method is to obtain a pseudo-yield stress below which the contribution of a pore size to the total flow rate is negligible.

The porous medium sample used in this study is a synthetic sintered silicate (Aerolith®10 purchased from PALL Corporation, USA) with a porosity and a permeability provided by the manufacturer of respectively $\epsilon=0.40$ and $K=5$ darcys.

\subsubsection{Experimental Setup and Operating Procedures}

A schematic representation of the experimental setup is displayed on Fig. 5. From left to right there are (1) a syringe pump filled alternatively with the polymer solution or the de-ionized water which ensures fluids injection at controlled flow rates, (2) the porous medium to be investigated, and (3) a vessel to collect the outgoing fluid. A pressure sensor is also connected to the inlet and the outlet of the porous media and the time evolution of the measured pressure drop was registered. To prepare the porous medium the following procedure was adopted: the $10 \mathrm{~cm}$ long sample was a cylindrical core of $5 \mathrm{~cm}$ diameter. The two extremities were in contact with aluminum injector plates. Surfaces in contact with the porous medium were Teflon (Du Pont, Washington, D.C., USA) coated to prevent any ion exchange between the metal and the core. The lateral surface of the porous medium was coated with a non-wetting epoxy resin then wrapped with epoxy-coated fiberglass to insure liquid imperviousness and mechanical strength.

Prior to the injection of xanthan gum solutions, the porous medium was saturated with degassed de-ionized and filtered water. Moreover, and to avoid any further bubble gas formation inside the porous medium, the polymer solution was degassed too under moderate vacuum. It should be finally noted that the temperature of the lab was kept constant at $T=20^{\circ} \mathrm{C} \pm 0.1$ during all the experiments. In a typical experiment, the imposed flow rate is steeply increased and the corresponding pressure drops are measured at the steady state 


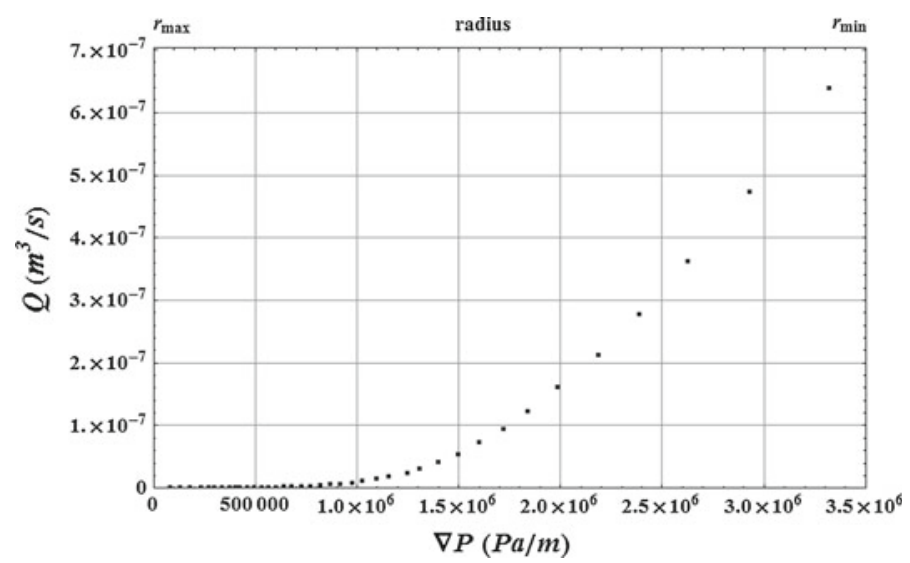

Fig. 6 Experimental $\left(Q_{i}, \nabla P_{i}\right)$ data obtained from the injection of a xanthan gum solution with $C_{\mathrm{p}}=$ 7,000 ppm through a sintered A10 silicate. The upper axis represents the radii of the pores joining the flow at each $\nabla P$ (smaller pores at high $\nabla P$ )

by starting at a flow rate that is the lowest provided by the pump in order to scan pores of the largest size. A sample of the outgoing fluid was characterized with the rheometer, and the obtained rheogram was found to be very close to the one of the fluid before injection. At the end of the experiment a set of $\left(Q_{i}, \nabla P_{i}\right)$ raw data are collected. Figure 6 shows the results obtained for $C_{\mathrm{p}}=7,000 \mathrm{ppm}$ with $N+1=39$. In any way, from this data set, firstly it is possible to determine the range of pore radii that may be explored by using Eq. (3) and subsequently the procedure described in Sect. 3 is used to obtain the PSD.

\subsubsection{Pore Size Distribution}

The method presented in Sect. 3 was applied to the raw data $\left(Q_{i}, \nabla P_{i}\right)$ obtained in the case of the investigated porous medium for $C_{\mathrm{p}}=7,000 \mathrm{ppm}$. The corresponding PSD expressed in terms of relative volume of pore classes of representative radii $r_{i}, p\left(r_{i}\right)$, is displayed on Fig. 7. The value of $\alpha^{*}$ obtained using the criterion presented by Eq. (9) was 1.3.

In the meantime the investigated porous medium was characterized by means of the classic mercury porosimetry and the obtained results are also presented in the same figure. Examination of this figure shows that both PSDs are approximately of Gaussian form. There is a good agreement between the PSD determined using the present method and the one obtained by MIP. It has been shown numerically that the value of $\alpha^{*}$ depends on the precision of the $(Q, \nabla P)$ measures. Hence, the observed difference between values of the current $\alpha^{*}=1.3$ and the one previously obtained for numerical experiments $\alpha^{*}=1.05$ may be attributed to the lower precision of the laboratory raw data. The fact that the fluid used is a pseudoplastic fluid without a true yield stress may also be considered as a factor of difference between the results from laboratory experiments and those from numerical experiments.

Furthermore and as in numerical experiments, a sensitivity analysis has been carried out so as to study the effect of the value of $\alpha$ on the obtained PSD. This is illustrated in Fig. 8. Once again, it is observed that $\alpha$ should be greater than one and that the MIP is best reproduced by choosing $\alpha=\alpha^{*}$ even if similar $\alpha$ values as in numerical experiments are used (1.05), acceptable PSDs are obtained despite some observed deviations. 


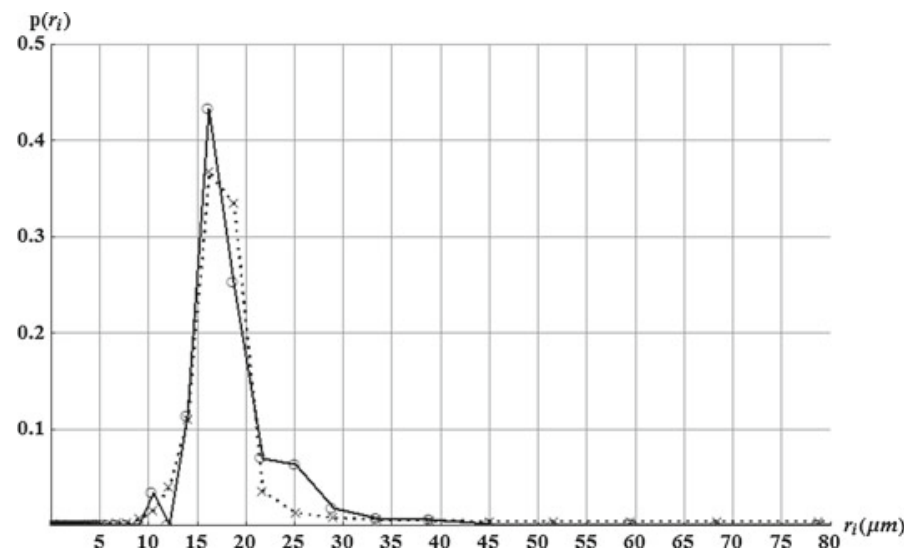

Fig. 7 PSD obtained with the present method corresponding to $C_{\mathrm{p}}=7,000 \mathrm{ppm}\left(\alpha^{*}=1.3\right)$ (circles joined by continuous lines) and comparison with PSD from MIP (crosses joined by dashed lines)

(a)

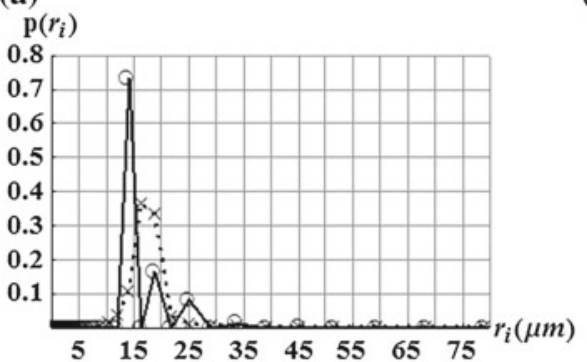

(c)

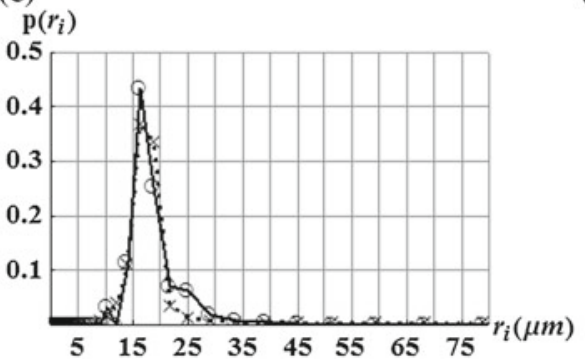

(b)

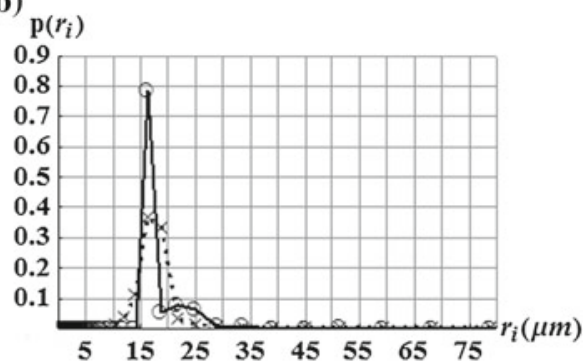

(d)

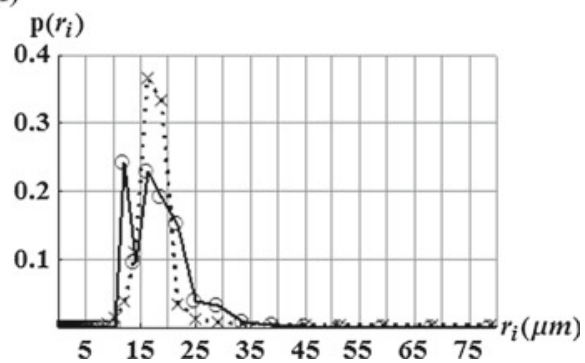

Fig. 8 PSD obtained with the present method corresponding to $C_{\mathrm{p}}=7,000 \mathrm{ppm}$ (circles joined by continuous lines) and comparison with PSD from MIP (crosses joined by dashed lines). $\mathbf{a} \alpha=1, \mathbf{b} \alpha=1.05, \mathbf{c} \alpha=1.3$, and $\mathbf{d} \alpha=1.5$

Complementary experiments were carried out under the same conditions and by changing only the xanthan concentration to $C_{\mathrm{p}}=6,000 \mathrm{ppm}$. In that case the obtained $\alpha^{*}$ is again 1.3 and the determined PSD is slightly shifted to small pore radii when compared to the PSD corresponding to $C_{\mathrm{p}}=7,000 \mathrm{ppm}$ (Fig. 9). This should be expected as when xanthan concentration is lowered the fluid rheology deviate more appreciably from the HerschelBulkley fluid type. This implies that the higher the polymer concentration, the better is the quality of the obtained PSDs. A sensitivity analysis concerning the effect of $\alpha$ was also carried out in this case $(6,000 \mathrm{ppm})$, concluding that $\alpha^{*}$ reproduced the best the MIP results (data not shown). 
(a)

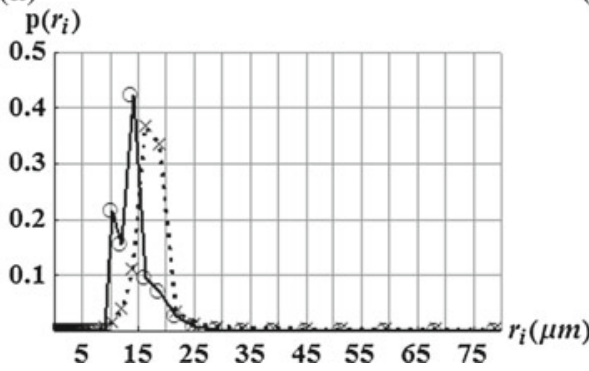

(b)

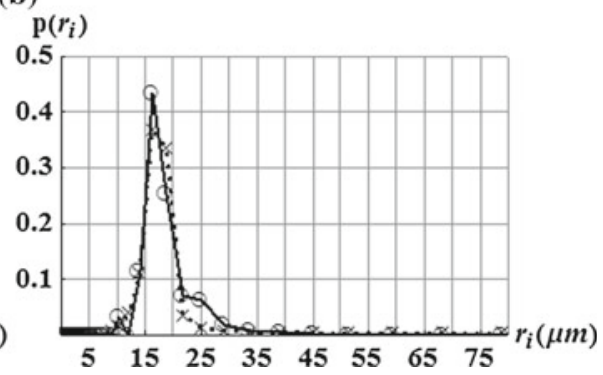

Fig. 9 PSD obtained with the present method corresponding to the two laboratory experiments (circles joined by continuous lines) and comparison with PSD from MIP (crosses joined by dashed lines). a $C_{\mathrm{p}}=6,000 \mathrm{ppm}$ $\left(\alpha^{*}=1.3\right), \mathbf{b} C_{\mathrm{p}}=7,000 \mathrm{ppm}\left(\alpha^{*}=1.3\right)$

Finally, it has to be taken into account that to assess the pertinence of the present method the MIP method is used as a reference which is obviously, as in our lab experiments, affected by several sources of error. In any way, it may be concluded that the method presented here can be considered as a new alternative for the derivation of the PSD of an unknown porous medium from simply measuring the change of flow rate versus the pressure gradient when a true or pseudo-yield stress fluid is injected through it.

\section{Discussion}

Some remarks should be addressed as follows:

(i) From a fundamental point of view, one may wonder which characteristic size of the actual pores is determined using the present method and what the relevance of the use of the PSD deduced from MIP is as a reference. To address these questions it is firstly reminded that the experiments carried out are essentially fluid transport experiments and, consequently, the pressure loss is mainly sensitive to hydrodynamic throat size rather than pore body size (Chauveteau February 2002). Consequently, the present method is not appropriate when one is interested in determining characteristics such as specific surface. Meanwhile, in the case of MIP, the PSD obtained from mercury intrusion measurements are dominated by pore throats, whereas pore bodies dominate in those calculated from mercury extrusion measurements. It is not then surprising that the PSD calculated with the present method are close to those coming from mercury intrusion. In general, PSD obtained by different methods may not be directly comparable. This aspect has to be kept in mind when comparing MIP results with other techniques such as tomography, whose results make a distinction between pore bodies size distribution and throats size distribution. Wardlaw et al. (1988) compared MIP distributions on carbonates with those obtained by means of an optical microscope finding a poor agreement between them, especially with regard to the small pores.

(ii) It is important to emphasize that the range of pore sizes that can be scanned with each porosimetry technique is restricted. This is of major importance when dealing with sedimentary rocks, whose pore sizes differ by several orders of magnitude. In that case, Radlinski et al. (2004) showed that the use of several techniques is needed to cover the whole range of pore radii from $20 \AA$ to $500 \mu \mathrm{m}$ in a sandstone from an oil reservoir in central Australia. 
(iii) Concerning yield stress fluids, they are generally complex media in which the dispersed objects are not negligibly small compared with smallest pores. So even if concentrated calibrated emulsions can be considered as good candidates, the minimum pore size that can be probed is typically of $300 \mathrm{~nm}$ (Mabille et al. 2000). Also, it is noticed that yield stress fluids such as Carbopol solutions (Islam et al. 2004; Kim et al. 2003; Tiu et al. 2006), alumina suspensions (Zhu and Smay 2011; Hirata et al. 2010), or bentonite suspensions (Ambrose and Loomis 1933) are not recommended because of their inherent thixotropy and viscoelasticity. Besides, other fluids than xanthan gum solutions should be used with porous media whose pore sizes are of the same order of magnitude as xanthan gum macromolecules to avoid pore-plugging phenomenon.

(iv) Some authors state that the yield stress does not truly exist (Barnes and Walters 1985) and is just an idealization due to the limited measurement time and experimental resolution. Careful inspection of the rheograms of xanthan gum solutions at low shear rates shows that, strictly speaking, there is no such yield stress. It is then reasonable to consider that the rheology of xanthan gum solutions is better represented by a Carreau model featuring a low shear viscosity plateau. However, and as it was put forward before, unfortunately no rigorous analytic relation expressing $Q$ versus $\nabla P$ in a capillary may be obtained. Therefore, the use of an asymptotic rheological law (truncated power law) should result in errors of the same order as those introduced using Herschel-Bulkley model. Moreover, to apply this model in the present method, it is necessary to define a "critical" viscosity above which a particular pore size class contributes only negligibly to the overall flow, and such "critical" viscosity would be analogous to a pseudo-yield stress. In any way, it is important that the shear-thinning character of the used fluid is sufficiently pronounced so that the fluid can be assimilated to a yield stress one (Sochi 2010). This is best accomplished in the case of concentrated polymer solutions.

(v) Real porous media are of course not bundles of capillaries and it is obvious that this model is not the most realistic one and is therefore unable to take into account nonlinear opening up of pores especially in the vicinity of the yield pressure gradient (Balhoff et al. 2012). This is a result of a complex percolation pattern only predictable by solving the pore-level physics (Balan et al. 2011). The use of more complex models for porous media such as pore networks would imply the necessity of knowing more details regarding the pore geometry and connectivity (coordination number, pore body-to-pore throat aspect ratio, etc.). This information is not available unless other techniques such as micro tomography and image analysis are used. Moreover, in these models a large but finite value of the viscosity is considered so as to emulate the yield stress (Sochi 2010). Despite recent efforts to simulate yield stress fluids flow through pore networks (Sochi and Blunt 2008; Balhoff and Thompson 2004), the resolution of the pore-level physics is not possible without knowing "a priori" the pore topology. The way to extend the present method to more representative models of porous media is to develop a relationship between the applied pressure gradient and the critical pore radius joining the flow at this pressure gradient. In the case of a bundle of capillaries model this relationship is given by Eq. (3). Other relationships could be found for pore networks through simulations as suggested by Sochi and Blunt (2008). The goal of the present paper is to propose a simple method that allows determination of representative PSD of real porous media without any input of topological parameters coming from other techniques. Finally, and as explained previously, the bundle of capillaries is also used in mercury porosimetry, so the present method will be "only" as bad as the mercury porosimetry concerning the representativeness of the porous morphology. 


\section{Conclusion}

A new method of PSD determination from yield stress fluids injection experiments is proposed. It is possible to get PSD from $Q(\nabla P)$ raw data from relatively easy to do experiments using straightforward calculations. The method has been successfully tested on numerical experiments and on two laboratory experiments. In the case of laboratory experiments it provides PSDs comparable to the mercury porosimetry results. Therefore, it may be concluded that the method presented here can be considered as a new alternative for the derivation of the PSD of a porous medium with unknown PSD. However, the reliability of this method has now to be tested over a wide range of porous medium samples with different permeabilities and pore sizes and adsorption phenomena have to be quantified and minimized. It should be noted that despite the number of questions raised, the first results presented here are very encouraging.

Acknowledgments Antonio Rodriguez de Castro wishes to thank La Caixa Foundation for its financial support through an international grant for postgraduate studies.

\section{References}

Abidina, A.Z., Puspasaria, T., Nugrohoa, W.A.: Polymers for enhanced oil recovery technology. Proc. Chem. 4, 11-16 (2012)

Ambari, A., Benhamou, M., Roux, S., Guyon, E.: Distribution des tailles des pores d'un milieu poreux déterminée par l'écoulement d'un fluide à seuil. C.R. Acad. Sci. Paris t.311(II), 1291-1295 (1990)

Ambrose, H.A., Loomis, A.G.: Fluidities of thixotropic gels: bentonite suspensions. Physics 4, 265 (1933)

Balan, H.O., Balhoff, M.T., Nguyen, Q.P., Rossen, W.R.: Network modeling of gas trapping and mobility in foam enhanced oil recovery. Energy Fuels 25(9), 3974-3987 (2011)

Balhoff, M.T., Thompson, K.E.: Modeling the steady flow of yield-stress fluids in packed beds. AlChE J. 50(12), 3034-3048 (2004)

Balhoff, M.T., Lake, L.W., Bommer, P.M., Lewis, R.E., Weber, M.J., Calderin, J.M.: Rheological and yield stress measurements of non-Newtonian fluids using a Marsh funnel. J. Petrol. Sci. Eng. 77(3-4), 393-402 (2011)

Balhoff, M., Sanchez-Rivera, D., Kwok, A., Mehmani, Y., Prodanovic, M.: Numerical algorithms for network modeling of yield stress and other non-Newtonian fluids in porous media. Transp. Porous Media 93(3), 363-379 (2012)

Barnes, H., Walters, K.: The yield stress myth? Rheol. Acta 24, 323-326 (1985)

Chaplain, V., Mills, P., Guiffant, G., Cerasi, P.: Model for the flow of a yield fluid through a porous medium. J. Phys. II France 2, 2145-2158 (1992)

Chauveteau, G.A.: The grain and pore throat model: a tool to predict mass transport and formation damage. In: SPE International Symposium and Exhibition on Formation Damage Control, Lafayette, Louisiana, pp. 20-21 (2002)

Clarkson, C.R., Solano, N., Bustin, R.M., Bustin, A.M.M., Chalmers, G.R.L., Hec, L., Melnichenko, Y.B., Radlinski, A.P., Blach, T.P.: Pore structure characterization of North American shale gas reservoirs using USANS SANS, gas adsorption, and mercury intrusion. Fuel 103, 606-616 (2013)

Galaup, S., Liu, Y., Cerepi, A.: New integrated 2D-3D physical method to evaluate the porosity and microstructure of carbonate and dolomite porous system. Microporous Mesoporous Mater. 154, 175-186 (2012)

Garcia-Ochoa, F., Santosa, V.E., Casasb, J.A., Gómez, E.: Xanthan gum: production, recovery, and properties. Biotechnol. Adv. 18, 549-579 (2000)

Giesche, H.: Mercury porosimetry: a general (practical) overview. Part. Part. Syst. Charact. 23, 1-11 (2006)

Hirata, Y., Matsushima, K., Matsunaga, N., Sameshima, S.: Viscoelastic properties of flocculated alumina suspensions during pressure filtration. J. Ceram. Soc. Jpn 118, 977-982 (2010)

Islam, M.T., Rodríguez-Hornedo, N., Ciotti, S., Ackermann, C.: Rheological characterization of topical carbomer gels neutralized to different pH. Pharma. Res. 21(7) (2004)

Kate, J.M., Gokhale, C.S.: A simple method to estimate complete pore size distribution of rocks. Eng. Geol. 84, 48-69 (2006)

Khodja, M.: Les fluides de forage: tude des performances et considerations environnementales. PhD thesis, Institut National Polytechnique de Toulouse (2008) 
Kim, J.-Y., Song, J.-Y., Lee, E.-J., Park, S.-K.: Rheological properties and microstructures of carbopol gel network system. Colloid Polym. Sci. 281, 614-623 (2003)

Lindquist, W.B., Venkatarangan, A.: Pore and throat size distributions measured from synchrotron X-ray tomographic images of Fontainebleau sandstones. J. Geophys. Res. Solid Earth 105(B9), 21509-21527 (2000)

Mabille, C., Schmitt, V., Gorria, P., Calderón, F.L., Faye, V., Deminière, B., Bibette, J.: Rheological and shearing conditions for the preparation of monodisperse emulsions. Langmuir 16, 422-429 (2000)

Mongruel, A., Cloitre, M.: Axisymmetric orifice flow for measuring the elongational viscosity of semi-rigid polymer solutions. J. Non-Newton. Fluid Mech. 110, 27-43 (2003)

Nguyen, Q., Boger, D.: Direct yield stress measurement with the vane method. J. Rheol. 29(3), 335-347 (1983)

Nimmo, J.R.: Porosity and pore size distribution. In: Hillel, D. (ed.) Encyclopedia of Soils in the Environment, vol. 3, pp. 295-303. Elsevier, London (2004)

Okabe, H., Blunt, M.J.: Pore space reconstruction using multiple-point statistics. J. Petrol. Sci. Eng. 46, 121-137 (2005)

Oukhlef, A.: Détermination de la distribution de tailles de pores d'un milieu poreux. PhD thesis, Arts et Métiers ParisTech-Centre Angers, Paris, 12 December (2011)

Oukhlef, A., Ambari, A., Champmartin, S., Despeyroux, A.: Détermination de la distribution de tailles de pores d'un milieu poreux par la méthode des fluides à seuil, 20ème Congrès Français de Mécanique, Besançon, 29 August-2 September (2011)

Palaniraj, A., Jayaraman, V.: Production, recovery and applications of xanthan gum by Xanthomonas campestris. J. Food Eng. 106, 1-12 (2011)

Plougonven, E.: Lien entre la microstructure des matériaux poreux et leur perméabilité. Mise en évidence des paramètres géométriques et topologiques influant sur les propriétés de transport par analyses dimages microtomographiques. PhD Thesis, Ecole Doctorale des Sciences Chimiques, Université de Bordeaux 1, 6 october (2009)

Prodanovic, M., Lindquist, W.B., Seright, R.S.: Porous structure and fluid partitioning in polyethylene cores from 3D X-ray microtomographic imaging. J. Colloid Interface Sci. 298, 282-297 (2006)

Prodanovic, M., Lindquist, W.B., Seright, R.S.: 3D image-based characterization of fluid displacement in a Berea core. Adv. Water Resourc. 30, 214-226 (2007)

Radlinski, A.P., Ioannidis, M.A., Hinde, A.L., Hainbuchner, M., Baron, M., Rauch, H., Kline, S.R.: Angstromto-millimeter characterization of sedimentary rock microstructure. J. Colloid Interface Sci. 274, 607-612 (2004)

Riche, F., Schneebeli, M., Tschanz, S.A.: Design-based stereology to quantify structural properties of artificial and natural snow using thin sections. Cold Reg. Sci. Technol. 79-80, 67-74 (2012)

Rouquerol, J., Baron, G.V., Denoyel, R., Giesche, H., Groen, J., Klobes, P., Levitz, P., Neimark, A.V., Rigby, S., Skudas, R., Sing, K., Thommes, M., Unger, K.: The characterization of macroporous solids: an overview of the methodology. Microporous Mesoporous Mater. 154, 2-6 (2012)

Skelland, A.H.P.: Non-Newtonian Flow and Heat Transfer. Wiley, New York (1967)

Sochi, T.: Modelling the flow of yield-stress fluids in porous media. Trans. Porous Med. 85, 489-503 (2010)

Sochi, T., Blunt, M.J.: Pore-scale network modeling of Ellis and Herschel-Bulkley fluids. J. Petrol. Sci. Eng. 60, 105-124 (2008)

Song, K.-W., Kim, Y.-S., Chang, G.S.: Rheology of concentrated xanthan gum solutions: steady shear flow behavior. Fibers Polym. 7(2), 129-138 (2006)

Sun, Z., Tang, X., Cheng, G.: Numerical simulation for tortuosity of porous media. Microporous Mesoporous Mater. 173, 37-42 (2013)

Tiu, C., Guo, J., Uhlherr, P.H.T.: Yielding behaviour of viscoplastic materials. J. Ind. Eng. Chem. 12(5), 653-662 (2006)

United Nations Environment Programme (UNEP).: Minamata convention on mercury. http://www. mercuryconvention.org/Convention/tabid/3426/Default.aspx (2013). Accessed 25 Oct 2013

Wadhai, V.S., Dixit, A.N.: Production of Xanthan gum by Xanthomonas campestris and comparative study of Xanthomonas campestris isolates for the selection of potential Xanthan producer. Indian Streams Res. J. 1, 1-4 (2011)

Wardlaw, N.C., McKellar, M., Yu, L.: Pore and throat size distributions determined by mercury porosimetry and by direct observation. Carbonates Evaporites 3, 1-16 (1988)

Weilbeer, M.: Efficient Numerical Methods for Fractional Differential Equations and Their Analytical Background. Papierflieger (2005).

Zhu, C., Smay, J.E.: Thixotropic rheology of concentrated alumina colloidal gels for solid freeform fabrication. J. Rheol. 55, 655-672 (2011) 